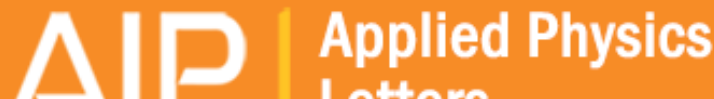 Letters
}

\section{Fermi level pinning by integer charge transfer at electrode-organic semiconductor interfaces}

Menno Bokdam, Deniz Çakır, and Geert Brocks

Citation: Applied Physics Letters 98, 113303 (2011); doi: 10.1063/1.3565963

View online: http://dx.doi.org/10.1063/1.3565963

View Table of Contents: http://scitation.aip.org/content/aip/journal/apl/98/11?ver=pdfcov

Published by the AIP Publishing

\section{Articles you may be interested in}

Mechanism of charge transfer and its impacts on Fermi-level pinning for gas molecules adsorbed on monolayer WS2

J. Chem. Phys. 142, 214704 (2015); 10.1063/1.4922049

Fermi level pinning induced electrostatic fields and band bending at organic heterojunctions

Appl. Phys. Lett. 105, 223303 (2014); 10.1063/1.4903360

Role of intrinsic molecular dipole in energy level alignment at organic interfaces

Appl. Phys. Lett. 102, 223301 (2013); 10.1063/1.4809567

Modeling charge transfer at organic donor-acceptor semiconductor interfaces

Appl. Phys. Lett. 100, 203302 (2012); 10.1063/1.4717985

Fermi-level pinning at conjugated polymer interfaces

Appl. Phys. Lett. 88, 053502 (2006); 10.1063/1.2168515

\section{AIP $\mid$ APL Photonics}

APL Photonics is pleased to announce Benjamin Eggleton as its Editor-in-Chief

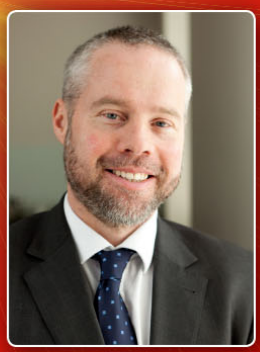




\title{
Fermi level pinning by integer charge transfer at electrode-organic semiconductor interfaces
}

\author{
Menno Bokdam, Deniz Çakır, and Geert Brocks ${ }^{a)}$ \\ Computational Materials Science, Faculty of Science and Technology and MESA+Institute for \\ Nanotechnology, University of Twente, P.O. Box 217, 7500 AE Enschede, The Netherlands
}

(Received 17 December 2010; accepted 23 February 2011; published online 14 March 2011)

\begin{abstract}
The atomic structure of interfaces between conducting electrodes and molecular organic materials varies considerably. Yet experiments show that pinning of the Fermi level, which is observed at such interfaces, does not depend upon the structural details. In this letter, we develop a general model to explain Fermi level pinning, and formulate simple expressions for the pinning levels, based upon integer charge transfer between the conductor and the molecular layer. In particular, we show that DFT calculations give good values for the pinning levels. (C) 2011 American Institute of Physics. [doi:10.1063/1.3565963]
\end{abstract}

Interfaces play an important role in organic light emitting diodes and solar cells. The Schottky barriers at the interfaces between conducting electrodes and organic semiconductors determine the injection and collection of charge carriers. ${ }^{1-3}$ In organic molecular semiconductors the molecules preserve their identity as they interact via relatively weak intermolecular (van der Waals) forces. Charge localization and polaron effects then play a much more important role than in conventional wide band semiconductors. ${ }^{4,5} \mathrm{Ex}$ perimentally the formation of Schottky barriers at electrodeorganic interfaces is dominated by the first molecular layer. Often these barriers are characterized by monitoring the work function of an organic layer deposited onto a metallic substrate through ultraviolet photoelectron spectroscopy (UPS). Such experiments in combination with first-principles calculations have lead to an impressive progress in understanding the interaction between well-ordered layers of organic molecules and single crystal metal surfaces, as well as to simple models for the resulting work functions. ${ }^{6-14}$

The contacts between electrodes and organic materials in actual devices are likely to be less well-defined than in these model systems, however. Metal electrodes are often covered by an (amorphous) native oxide layer and conducting oxides or polymers, such as indium tin oxide (ITO) or poly $(3,4-$ ethylenedioxythiophene) (PEDOT), are commonly amorphous. UPS experiments on interfaces between such electrodes and organic materials show nevertheless a universal behavior. For substrates with work functions within a certain energy interval the organic layer changes the work function depending on the details of the interaction between molecules and substrate (such as the pillow or pushback effect). Remarkably, outside this interval the work function is completely pinned, i.e., independent of the substrate work function, see Fig. 1. ${ }^{3}$ All substrates with a work function lower than $W^{-}$lead to pinning at $W^{-}$, and all substrates with a work function higher than $W^{+}$lead to pinning at $W^{+}$. The pinning levels seem to be related to the electron affinity $(A)$ and the ionization potential $(I)$ of the molecules in the layer, respectively. However, measured values of $W^{-/+}$differ from $A$ or $I$ by up to $\sim 1 \mathrm{eV}$. Such "shifts" have been ascribed to polaron

\footnotetext{
${ }^{\text {a) }}$ Author to whom correspondence should be addressed. Electronic mail: g.brocks@tnw.utwente.nl.
}

effects but seem far too large to originate from molecular relaxation only.

Here we develop and test a model for Fermi level pinning at electrode-organic semiconductor interfaces. We will argue that pinning occurs by transfer of electrons to or from the molecular levels, and that the shifts observed experimentally mainly originate from the electrostatic interactions at the interface, which lower the energy. For an arbitrary charge distribution this would lead to a shift that depends on the amount of charge transfer but if the interfacial charge distribution can be described by a plane capacitor, the shift is constant. The lower and upper pinning levels $W^{-/+}$, see Fig. 1 are then given by

$$
W^{-}=A+B^{-}, \quad W^{+}=I-B^{+},
$$

where $B^{-/+}$is the Coulomb energy associated with charging a molecule in the interface layer with an electron/hole. The individual parameters $A, B, I$ depend on the environment of the molecule, as charged molecules are screened by their environment. As is discussed below, such screening terms
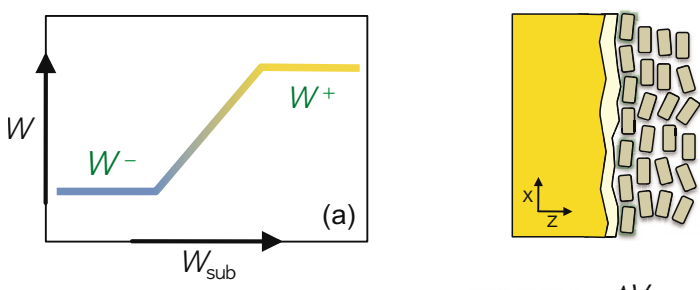

(b)

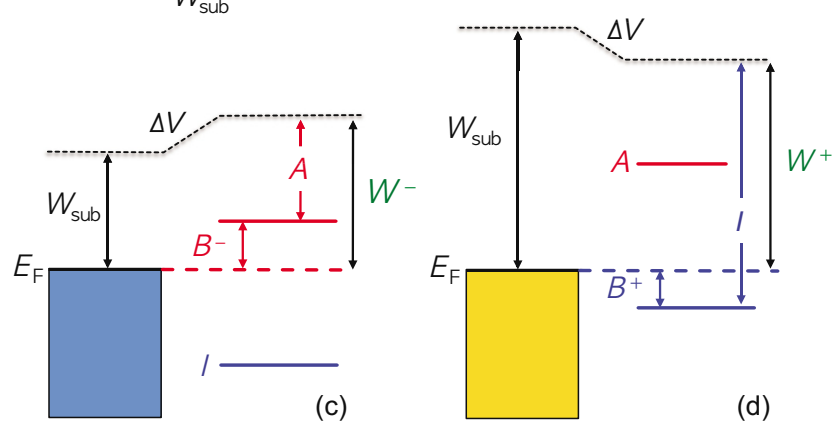

FIG. 1. (Color online) (a) The work function $W$ of the electrode-organic interface (b) as function of the electrode work function $W_{\text {sub. The constants }}$ $W^{-/+}$indicate Fermi level pinning on low/high work function substrates, according to level diagrams (c)/(d), respectively. 
cancel in $W^{-/+}$, which implies that the Fermi level is pinned and that the values of the pinning levels are a property of the molecular layer only. For example, for C60 layers adsorbed on different low work function substrates a pinning level is observed at $W^{-}=4.5 \mathrm{eV} .{ }^{15}$ The difference between this value and the electron affinity $A=4.0 \mathrm{eV}$ found for C60 layers, is in good agreement with the charging energy $B^{-}$ $=0.55 \pm 0.15 \mathrm{eV}$ of a C60 molecule in a layer. ${ }^{16}$

In principle the parameters $A, B, I$ can be extracted from experiment or from calculations. For density functional theory (DFT) calculations with standard generalized gradient approximation (GGA) functionals additional simplifications are possible, yielding very simple approximations for the pinning levels

$$
W^{-}=-\epsilon_{\mathrm{LUMO}}+E_{\mathrm{rel}}^{-} ; \quad W^{+}=-\epsilon_{\mathrm{HOMO}}-E_{\mathrm{rel}}^{+},
$$

where $\epsilon_{\text {HOMO/Lumo }}$ are the DFT (Kohn-Sham) energy levels of the highest occupied/lowest unoccupied molecular orbitals (HOMO/LUMO) of the neutral molecular layer. $E_{\mathrm{rel}}^{-++}$are the energies associated with structural relaxation of the molecule upon charging it with an electron or hole, or in other words, the energies attributed to polaron effects. The latter turn out to be relatively small for the molecules we have tested.

Pinning can only take place if a potential step $\Delta V$ occurs at the interface, see Fig. 1. If there is no significant hybridization between the substrate and the molecular wave functions, a potential step must be the result of transfer of an integer number of electrons between the substrate and the molecular layer. We derive a simple model for such integer charge transfer of electrons from the substrate to a layer of acceptor molecules, resulting in an expression for $W^{-3}$. Deriving an expression for $W^{+}$, which involves electron transfer from donor molecules to the substrate, then proceeds along the same lines.

The work function of a substrate covered by a molecular layer is given by

$$
W=-E_{\mathrm{F}}+\Delta V\left(N_{1}\right),
$$

where $E_{\mathrm{F}}$ is the Fermi level of the metal $\left(-E_{\mathrm{F}}\right.$ is the work function of the substrate without the molecular layer). $\Delta V\left(N_{1}\right)$ is the potential step caused by the $N_{1}$ charges in the molecular layer and the screening charges in the substrate. The details of the charge distribution do not matter for the work function, as the latter is measured far from the interface, compared to the molecular dimensions. ${ }^{17}$ One can express the potential step in terms of a dipole density

$$
\Delta V\left(N_{1}\right)=\frac{e D}{\varepsilon_{0} a} \frac{N_{1}}{N}
$$

with $D$ is a dipole associated with each charged molecule and its image charge, $a$ the surface area covered by one neutral or charged molecule, and $N$ the total number of molecules. Defining an interface capacitance by $C=e \varepsilon_{0} a N / D$ allows one to rewrite $\Delta V$ in plane capacitor form

$$
\Delta V\left(N_{1}\right)=\frac{N_{1} e^{2}}{C} .
$$

The total energy of a layer of $N_{1}$ charged molecules and $N-N_{1}$ neutral molecules is

$$
E_{\text {tot }}\left(N_{1}\right)=\left(N-N_{1}\right) E_{0}+N_{1} E_{1}+E_{\mathrm{C}}\left(N_{1}\right),
$$

where $E_{0}$ and $E_{1}$ are the total energies of a neutral and a charged molecule, respectively. $E_{\mathrm{C}}\left(N_{1}\right)$ is the electrostatic Coulomb energy of the interfacial arrangement of the charged molecules, all polarization and image charge effects included. If we assume that the electrostatic energy of the interface is described well by a plane capacitor with a capacitance as in Eq. (5), then

$$
E_{\mathrm{C}}\left(N_{1}\right)=\frac{N_{1}^{2} e^{2}}{2 C}-N_{1} B^{-} .
$$

Here $B^{-}$is the Coulomb energy associated with charging a single molecule. It has to be subtracted from the plane capacitor charging energy to avoid double counting, as per definition it is already accounted for in $E_{1}$, see Eq. (6). The electro-chemical potential of the ensemble of molecules (at $T=0)$ is given by $\mu=E_{\text {tot }}\left(N_{1}+1\right)-E_{\text {tot }}\left(N_{1}\right)$ in the thermodynamic limit $N_{1} \gg 1$. At equilibrium $\mu$ is equal to $E_{\mathrm{F}}$, the Fermi level of the substrate, which fixes the number of charged molecules $N_{1}$. Using this in Eqs. (5) and (3), and the definition of the electron affinity $A=E_{0}-E_{1}$, then leads to $W^{-}$ as in Eq. (1); $W^{+}$is derived in a similar way.

Note that the Fermi level (or work function) of the substrate, as well as the capacitance $C$ of the interface, drop out of the expression. One might argue that Eq. (1) still does not represent work function pinning, as all individual parameters $A, I$ and $B^{-/+}$strongly depend on the environment of the molecule, including the substrate. Screening by an environment stabilizes a charged molecule, thereby increasing its electron affinity and decreasing its ionization potential. Replacing a substrate alters the screening energy by $\Delta E_{\mathrm{S}}$ and changes the electron affinity and ionization potential to $A^{\prime}=A+\Delta E_{\mathrm{S}}$ and $I^{\prime}=I-\Delta E_{\mathrm{S}}$, respectively. However, at the same time the charging energy of a molecule is changed to $B^{\prime}=B-\Delta E_{\mathrm{S}}$, which shows that the values of $W^{-/+}$obtained from Eq. (1) are independent of the substrate. In other words, we have work function pinning and the pinning levels are a property of the molecular layer only.

The parameters $A, I$ and $B^{-/+}$are accessible from (firstprinciples) calculations on isolated molecular layers. ${ }^{18}$ In particular for DFT calculations with GGA or LDA functionals further simplifying approximations are possible. Those functionals yield an expression of the molecular total energy that is analytical in the occupation numbers $n_{i}$ of the KohnSham energy levels $\varepsilon_{i}$. Janak's theorem holds for such functionals, which expresses the energy levels as derivatives of the total energy $\varepsilon_{i}=\partial E_{\mathrm{mol}} / \partial n_{i}$. The vertical electron affinity can then be approximated by

$$
A_{\mathrm{v}}=-\int_{0}^{1} \varepsilon_{\mathrm{LUMO}}(n) d n \approx-\epsilon_{\mathrm{LUMO}}-\frac{1}{2} U,
$$

assuming that a linear approximation $\varepsilon_{\mathrm{LUMO}}(n)=\epsilon_{\mathrm{LUMO}}$ $+U n$ is sufficiently accurate. Here $\epsilon_{\text {LUMO }}$ is the Kohn-Sham LUMO level of a neutral molecule in the molecular layer. Integrating Janak's expression in the same approximation gives $E_{\mathrm{mol}}(n)=E_{0}+\epsilon_{\mathrm{LUMO}} n+1 / 2 U n^{2}$ for the total energy of a molecule with a partially occupied LUMO level. The quadratic term can be associated with the molecular charging energy 
TABLE I. Calculated relaxation energies, pinning levels according to Eq. (2), and experimental pinning levels; all in eV. Pinning is to the LUMO, i.e., $W^{-}$, unless indicated otherwise.

\begin{tabular}{lccc}
\hline \hline Molecule & $E_{\text {relax }}$ & $W$ calc. & $W$ expt. \\
\hline F4-TCNQ & 0.10 & 5.7 & $5.6^{\mathrm{a}}$ \\
TCNQ & 0.10 & 5.4 & $4.8^{\mathrm{b}}$ \\
PTCDA & 0.09 & 4.7 & $4.7^{\mathrm{c}}$ \\
C60 & 0.05 & 4.4 & $4.5^{\mathrm{d}}$ \\
CuPc & 0.05 & 3.3 & $3.3^{\mathrm{e}}$ \\
CuPc $^{\mathrm{f}}$ & 0.02 & 4.4 & $4.4^{\mathrm{g}}$ \\
TTF $^{\mathrm{f}}$ & 0.10 & 4.2 & $4.2^{\mathrm{b}}$ \\
\hline \hline
\end{tabular}

${ }^{\mathrm{a}}$ Reference 22.

${ }^{\mathrm{b}}$ Reference 23.

${ }^{c}$ Reference 24.

${ }^{\mathrm{d}}$ Reference 15 .

${ }^{\mathrm{e}}$ Reference 25.

${ }^{\mathrm{f}}$ Pinning to the HOMO, i.e., $W^{+}$.

${ }^{\mathrm{g}}$ Reference 26 .

$$
B^{-} \approx \frac{1}{2} U
$$

Equation (1) requires the adiabatic $A$ and $I$, which can be obtained from the corresponding vertical properties by correcting with the molecular relaxation energies, i.e., $A=A_{\mathrm{v}}$ $+E_{\mathrm{rel}}^{-}$and $I=I_{\mathrm{v}}+E_{\mathrm{rel}}^{+}$. Using Eqs. (8) and (9) then gives Eq. (2).

We calculate Kohn-Sham energy levels using the Vienna $A b$ initio Simulation Package (VASP) with projector augmented waves and the PW91 GGA functional. ${ }^{19-21}$ Calculations using the PBE functional yield pinning levels that are within $0.05 \mathrm{eV}$ of the values presented in this paper. The calculations are performed for close-packed molecular monolayers. The unit cell in the direction perpendicular to that plane is chosen sufficiently large that the potential in the middle of the cell represents the vacuum level. The KohnSham energy levels are then calculated with respect to this vacuum level.

The pinning levels calculated according to Eq. (2) are given in Table I. The calculated values are in good agreement with available experimental data, indicating that the essential physics is grasped by our simple model. TCNQ seems to be an exception, where the calculation overestimates the value of the pinning level to the LUMO. Note that the relaxation energies play a relatively unimportant role in fixing the pinning levels, as they are quite small. The energy difference between the upper and the lower pinning levels $W^{+}-W^{-}$for $\mathrm{CuPc}$ is less than half the band gap in this material. ${ }^{27}$ Whereas our model gives a natural explanation for this ef- fect, it would be difficult to explain this on the basis of molecular relaxation only.

This work is part of the European project MINOTOR, Grant No. FP7-NMP-228424.

${ }^{1}$ H. Ishii, K. Sugiyama, E. Ito, and K. Seki, Adv. Mater. (Weinheim, Ger.) 11, 605 (1999)

${ }^{2}$ A. Kahn, N. Koch, and W. Gao, J. Polym. Sci., B, Polym. Phys. 41, 2529 (2003)

${ }^{3}$ S. Braun, W. R. Salaneck, and M. Fahlman, Adv. Mater. (Weinheim, Ger.) 21, 1450 (2009).

${ }^{4}$ G. Brocks, Synth. Met. 102, 914 (1999).

${ }^{5}$ G. Brocks, J. van den Brink, and A. F. Morpurgo, Phys. Rev. Lett. 93, 146405 (2004).

${ }^{6}$ H. Vázquez, R. Oszwaldowski, P. Pou, J. Ortega, R. Pérez, F. Flores, and A. Kahn, Europhys. Lett. 65, 802 (2004).

${ }^{7}$ H. Vázquez, F. Flores, R. Oszwaldowski, J. Ortega, R. Pérez, and A. Kahn, Appl. Surf. Sci. 234, 107 (2004).

${ }^{8}$ L. Romaner, G. Heimel, J.-L. Brédas, A. Gerlach, F. Schreiber, R. L. Johnson, J. Zegenhagen, S. Duhm, N. Koch, and E. Zojer, Phys. Rev. Lett. 99, 256801 (2007).

${ }^{9}$ S. Duhm, G. Heimel, I. Salzmann, H. Glowatzki, R. L. Johnson, A. Vollmer, J. Rabe, and N. Koch, Nature Mater. 7, 326 (2008).

${ }^{10}$ G. M. Rangger, O. T. Hofmann, L. Romaner, G. Heimel, B. Bröker, R.-P. Blum, R. L. Johnson, N. Koch, and E. Zojer, Phys. Rev. B 79, 165306 (2009).

${ }^{11}$ P. C. Rusu, G. Giovannetti, C. Weijtens, R. Coehoorn, and G. Brocks, J. Phys. Chem. C 113, 9974 (2009).

${ }^{12}$ P. C. Rusu, G. Giovannetti, C. Weijtens, R. Coehoorn, and G. Brocks, Phys. Rev. B 81, 125403 (2010).

${ }^{13}$ L. Vitali, G. Levita, R. Ohmann, A. Comisso, A. D. Vita, and K. Kern, Nature Mater. 9, 320 (2010).

${ }^{14}$ T.-C. Tseng, C. Urban, Y. Wang, R. Otero, S. L. Tait, M. Alcamí, D. Écija, M. Trelka, J. M. Gallego, N. Lin, M. Konuma, U. Starke, A. Nefedov, A. Langner, C. Wöll, M. Á. Herranz, F. Martín, N. Martín, K. Kern, and R. Miranda, Nature Chem. 2, 374 (2010).

${ }^{15}$ W. Osikowicz, M. P. de Jong, and W. R. Salaneck, Adv. Mater. (Weinheim, Ger.) 19, 4213 (2007).

${ }^{16}$ O. Gunnarsson, Rev. Mod. Phys. 69, 575 (1997).

${ }^{17}$ D. Cahen and A. Kahn, Adv. Mater. (Weinheim, Ger.) 15, 271 (2003).

${ }^{18}$ The fact that screening does not affect the pinning level does not mean that it is a purely molecular property, however. Electrostatic molecular multipoles can affect the energy levels of a molecule in a layer, see Ref. 9.

${ }^{19}$ G. Kresse and J. Hafner, Phys. Rev. B 47, 558(R) (1993).

${ }^{20}$ G. Kresse and J. Furthmüller, Phys. Rev. B 54, 11169 (1996).

${ }^{21}$ G. Kresse and D. Joubert, Phys. Rev. B 59, 1758 (1999).

${ }^{22}$ S. Braun and W. R. Salaneck, Chem. Phys. Lett. 438, 259 (2007).

${ }^{23}$ R. J. Murdey and W. R. Salaneck, Jpn. J. Appl. Phys., Part 1 44, 3751 (2005).

${ }^{24}$ I. Hill, D. Milliron, J. Schwartz, and A. Kahn, Appl. Surf. Sci. 166, 354 (2000).

${ }^{25}$ Y. Tanaka, K. Kanai, Y. Ouchi, and K. Seki, Org. Electron. 10, 990 (2009).

${ }^{26}$ H. Peisert, A. Petr, L. Dunsch, T. Chassé, and M. Knupfer, ChemPhysChem 8, 386 (2007).

${ }^{27}$ G. Giovannetti, G. Brocks, and J. van den Brink, Phys. Rev. B 77, 035133 (2008) 